\title{
Changes in Cardiopulmonary Reserve and Peripheral Arterial Function Concomitantly with Subclinical Inflammation and Oxidative Stress in Patients with Heart Failure with Preserved Ejection Fraction
}

\author{
Damien Vitiello, ${ }^{1,2}$ François Harel, ${ }^{1,2}$ Rhian M. Touyz, ${ }^{3,4}$ Martin G. Sirois, ${ }^{1,2}$ \\ Joel Lavoie, ${ }^{1}$ Jonathan Myers, ${ }^{5}$ Anique Ducharme, ${ }^{1}$ Normand Racine, ${ }^{1}$ \\ Eileen O'Meara, ${ }^{1}$ Mathieu Gayda, ${ }^{1,2,6}$ Malorie Chabot-Blanchet, ${ }^{7}$ Jean Lucien Rouleau, ${ }^{1}$ \\ Simon de Denus, ${ }^{1,8}$ and Michel White ${ }^{1,2}$ \\ ${ }^{1}$ Research Center, Montreal Heart Institute, Université de Montréal, 5000 Belanger Street East, \\ Montreal, QC, Canada H1T 1C8 \\ ${ }^{2}$ Departments of Medicine and Pharmacology, Faculty of Medicine, Université de Montréal, Montreal, QC, Canada \\ ${ }^{3}$ Institute of Cardiovascular and Medical Sciences, University of Glasgow, Glasgow, UK \\ ${ }^{4}$ The Kidney Research Center, Ottawa Hospital Research Institute, University of Ottawa, Ottawa, ON, Canada \\ ${ }^{5}$ Palo Alto VA Health Care System, Stanford University, Palo Alto, CA, USA \\ ${ }^{6}$ Cardiovascular Prevention and Rehabilitation Centre, Montreal Heart Institute, Montreal, QC, Canada \\ ${ }^{7}$ Coordinating Center, Montreal Heart Institute, Montreal, QC, Canada \\ ${ }^{8}$ Faculty of Pharmacy, Université de Montréal, Montreal, QC, Canada
}

Correspondence should be addressed to Michel White; m_white@icm-mhi.com

Received 25 July 2013; Accepted 14 January 2014; Published 27 February 2014

Academic Editor: Robert M. Schainfeld

Copyright () 2014 Damien Vitiello et al. This is an open access article distributed under the Creative Commons Attribution License, which permits unrestricted use, distribution, and reproduction in any medium, provided the original work is properly cited.

Background. Changes in cardiopulmonary reserve and biomarkers related to wall stress, inflammation, and oxidative stress concomitantly with the evaluation of peripheral arterial blood flow have not been investigated in patients with heart failure with preserved ejection fraction (HFpEF) compared with healthy subjects (CTL). Methods and Results. Eighteen HFpEF patients and 14 CTL were recruited. Plasma levels of inflammatory and oxidative stress biomarkers were measured at rest. Brain natriuretic peptide (BNP) was measured at rest and peak exercise. Cardiopulmonary reserve was assessed using an exercise protocol with gas exchange analyses. Peripheral arterial blood flow was determined by strain gauge plethysmography. Peak $\mathrm{VO}_{2}(12.0 \pm 0.4$ versus $19.1 \pm 1.1 \mathrm{~mL} / \mathrm{min} / \mathrm{kg}, P<0.001)$ and oxygen uptake efficiency slope $(1.55 \pm 0.12$ versus $2.06 \pm 0.14, P<0.05)$ were significantly decreased in HFpEF patients compared with CTL. BNP at rest and following stress, C-reactive-protein, interleukin-6, and TBARS were significantly elevated in HFpEF. Both basal and posthyperemic arterial blood flow were not significantly different between the HFpEF patients and CTL. Conclusions. HFpEF exhibits a severe reduction in cardiopulmonary reserve and oxygen uptake efficiency concomitantly with an elevation in a broad spectrum of biomarkers confirming an inflammatory and prooxidative status in patients with HFpEF.

\section{Introduction}

Heart failure with preserved ejection fraction (HFpEF) is associated with a decrease in cardiopulmonary reserve leading to significant maladaptive changes in peripheral arterial [1] and muscular functions [2]. Cardiopulmonary reserve and oxygen uptake efficiency are both decreased in chronic heart failure with reduced ejection fraction (HF-rEF) 
$[3,4]$. Other small studies have demonstrated that the oxygen uptake efficiency slope (OUES) is decreased in chronic HF patients [5] and in older patients with HFpEF [6].

HFpEF is characterized by an increase in some biomarkers related to neurohumoral activation $[7,8]$. Previous investigations have reported significant differences between patients with HFpEF versus HF patients with reduced ejection fraction $[7,8]$ such as lower N-terminal prohormone of brain natriuretic peptide (NT-proBNP) in HFpEF. The characterization of changes in biomarkers at rest and following peak exercise has not been fully addressed in this form of HF. Similarly, disorders of endothelial function and peripheral arterial blood flow have been a matter of controversies in patients with HFpEF [1,9-12]. No investigations have studied the changes in biomarkers related to LV wall stress, subclinical inflammation, and oxidative stress concomitantly with the evaluation of cardiopulmonary reserve and peripheral arterial function in HFpEF compared with healthy subjects.

The primary objective of this study was to investigate the changes cardiopulmonary reserve and peripheral arterial function, and biomarkers related to neurohumoral activation, inflammation, and oxidative stress in patients with HFpEF compared with healthy subjects. The secondary objective was to explore the relationship between biomarkers and functional capacity.

\section{Methods}

2.1. Study Population. This study was a prospective nonrandomized investigation including both patients with HFpEF and healthy subjects. Eighteen (18) patients and 14 healthy subjects were recruited. Patients were included in the HFpEF group if they had New York Heart Association (NYHA) classes II and III symptoms and if they had a left ventricle ejection fraction (LVEF) $\geq 50 \%$ measured by echocardiography within the 12 months prior to enrolment in the study. The diagnosis of HFpEF was confirmed by the presence of at least one abnormality on the screening echocardiography consistent with this condition such as atrial dilatation, left ventricle (LV) concentric remodeling or hypertrophy, and/or evidence of diastolic dysfunction by Doppler studies. LV volumes and filling rates were further assessed by radionuclide ventriculography at the beginning of the study. Patients with symptomatic hypotension (systolic blood pressure $(\mathrm{SBP})<$ $90 \mathrm{mmHg}$ ) or poorly controlled hypertension (SBP $\geq 160$ and/or diastolic blood pressure $>90 \mathrm{mmHg}$ ) were excluded. Similarly, patients with severe chronic pulmonary disease limiting exercise capacity, severe renal failure (creatinine $>$ $250 \mu \mathrm{mol} / \mathrm{L}$ ), or significant liver dysfunction (transaminases $\geq 3$-fold upper normal values) were excluded. Healthy subjects were included if they presented with no significant medical conditions and were on no medication at the time of assessment. Subjects or patients presenting with acute or active chronic inflammatory conditions were excluded from this study. All patients and healthy subjects provided written informed consent before undergoing any studyrelated procedures. The investigation conforms to the principles outlined in the Declaration of Helsinki. The study was approved by the Montreal Heart Institute-Research Scientific and Ethics Committees.

2.2. Maximal Exercise Testing. The maximal exercise test was performed on a treadmill using a RAMP protocol [13]. Gas exchange parameters were measured breath by breath during testing, and then averaged every 15 seconds for minute ventilation ( $\mathrm{VE}, \mathrm{L} / \mathrm{min}), \mathrm{O}_{2}$ uptake $\left(\mathrm{VO}_{2}, \mathrm{~L} / \mathrm{min}\right)$, and $\mathrm{CO}_{2}$ production $\left(\mathrm{VCO}_{2}, \mathrm{~L} / \mathrm{min}\right)$ using an automated gas analyzer system (Oxycon Pro, Hoechberg, Germany) [14]. Heart rate and manual brachial blood pressure were recorded before the test and at 2-minute intervals during exercise and recovery. Criteria for maximal effort were the attainment of the primary maximal criteria, a leveling off of oxygen uptake $(<150 \mathrm{~mL} / \mathrm{min})$ despite increased intensity or one of the three secondary maximal criteria: (1) a respiratory exchange ratio $>1.05$, (2) inability to maintain walking, and (3) patient exhaustion due to fatigue or other clinical symptoms (dyspnea, ECG, and/or blood pressure abnormalities) [14]. The average value of the $\mathrm{VO}_{2}$ recorded during the last 15 seconds of exercise was considered as the peak oxygen uptake $\left(\mathrm{VO}_{2}\right.$ peak), and $\mathrm{VE} / \mathrm{VCO}_{2}$ slope was also determined. The oxygen uptake efficiency slope (OUES) was calculated during exercise using the slope of the relation $\mathrm{VO}_{2}$ and the log of ventilation as previously reported [15]. The heart rate recovery (HRR) was measured at 1 (HRR 1 ) and 2 (HRR 2) minutes following the termination of exercise.

2.3. Biomarkers Measurements. Venous blood samples were taken after semisupine rest for at least 15 minutes from both experimental populations under fasting state in the morning. Serum samples were centrifuged $\left(1500 \mathrm{~g}, 15 \mathrm{~min}, 4^{\circ} \mathrm{C}\right)$ and immediately frozen at $-80^{\circ} \mathrm{C}$. Blood tests were performed in the resting state for all parameters and within 2 minutes following peak exercise for the brain natriuretic peptide (BNP).

Neurohumoral activation was assessed by plasma levels of both BNP and NT-proBNP. These two biomarkers were measured by electrochemiluminescence immunoassay using the Roche BNP and proBNP assays (Roche Diagnostics, Mannheim, Germany) on the Elecsys 2010 analyzer (Roche Diagnostics). Serum high-sensitivity C-reactive protein (hsCRP) was measured using the Dade Behring CardioPhase hsCRP assay (Siemens Healthcare Diagnostics Products, Marburg, Germany) on the BN ProSpec Nephelometer (Siemens Healthcare Diagnostics Products). Plasma level of thiobarbituric acid reactive substances (TBARS) was measured colorimetrically as previously described [16]. Plasma levels of interleukin-6 (IL-6) and 8-epi-prostaglandin F2 $\alpha$ were analyzed by ELISA using the R\&D Systems kits (Minneapolis, MN, USA).

2.4. Strain Gauge Plethysmography (SGP). All measurements of blood flow were performed 2 hours after morning medications. Forearm basal arterial flow was assessed using the strain gauge plethysmography (SGP) methods as previously described [17]. Briefly, all subjects sat with their arms resting in a supine position on supports positioned above the level of the heart. Venous cuffs were then connected to automatic 
TABLE 1: Baseline characteristics of the study population.

\begin{tabular}{|c|c|c|}
\hline Clinical variables & $\begin{array}{l}\text { HFpEF patients } \\
\quad(n=18)\end{array}$ & $\begin{array}{l}\text { Healthy controls } \\
\quad(n=14)\end{array}$ \\
\hline Age (years) & $70.7 \pm 8.9^{*}$ & $61.7 \pm 9.9$ \\
\hline Male & $5(28 \%)$ & $6(43 \%)$ \\
\hline Heart rate $(\mathrm{bpm})$ & $60.8 \pm 8.9^{*}$ & $70.2 \pm 7.7$ \\
\hline Systolic blood pressure (mmHg) & $125 \pm 16$ & $126 \pm 18$ \\
\hline Diastolic blood pressure (mmHg) & $72.4 \pm 8.2$ & $76.3 \pm 7.1$ \\
\hline Duration of heart failure (months) & $22.3 \pm 24.2$ & - \\
\hline \multicolumn{3}{|l|}{ NYHA functional class } \\
\hline II & $15(83 \%)$ & $0(0 \%)$ \\
\hline III & $3(17 \%)$ & $0(0 \%)$ \\
\hline \multicolumn{3}{|l|}{ Etiology of heart failure } \\
\hline Ischemic & $3(17 \%)$ & $0(0 \%)$ \\
\hline Hypertension & $15(83 \%)$ & $0(0 \%)$ \\
\hline \multicolumn{3}{|l|}{ Laboratory values } \\
\hline Haemoglobin (mg/L) & $131 \pm 13^{* *}$ & $145 \pm 12$ \\
\hline Serum creatinine $(\mu \mathrm{mol} / \mathrm{L})$ & $106 \pm 43^{*}$ & $79.7 \pm 15.4$ \\
\hline \multicolumn{3}{|l|}{ Medications } \\
\hline ACE inhibitors & $1(6 \%)$ & $0(0 \%)$ \\
\hline ARBs & $12(67 \%)$ & $0(0 \%)$ \\
\hline Beta-blockers & $9(50 \%)$ & $0(0 \%)$ \\
\hline \multicolumn{3}{|l|}{ Radionuclide angiography } \\
\hline LVEF (\%) & $57.5 \pm 7.0^{*}$ & $52.1 \pm 6.2$ \\
\hline LVEDV (mL) & $118.3 \pm 33.3^{*}$ & $98.0 \pm 19.1$ \\
\hline PFR (EDV/s) & $1.95 \pm 0.50^{*}$ & $2.34 \pm 0.42$ \\
\hline TPFR (ms) & $182 \pm 53^{*}$ & $147 \pm 40$ \\
\hline
\end{tabular}

ACE: angiotensin-converting enzyme; ARBs: angiotensin II receptor blockers; LVEDV: left ventricle end-diastolic volume; PFR: peak filling rate of the left ventricle; TPFR: time to peak filling rate of the left ventricle; LVEF: left ventricular ejection fraction; NYHA: New York Heart Association. Continuous variables are expressed as mean \pm standard deviation and categorical variables as frequencies and percentages. ${ }^{*} P<0.05 ;{ }^{* *} P<0.01$.

pneumatic inflators (Hokanson, E-20 rapid cuff inflator; Bellevue, WA) set to $50 \mathrm{mmHg}$ and calibrated strain gauges were placed around both forearms and connected to a plethysmograph (Hokanson, model EC-4, Bellevue, WA). Baseline flow measurements were performed before and after a 240-second period of arterial occlusion. Arterial inflow was calculated by determining the upslope of strain gauge signals calculated using a linear regression model.

2.5. Statistical Analyses. Continuous baseline characteristics are expressed as mean \pm standard deviation and categorical variables as frequencies and percentages. A logarithmic transformation was applied to variables showing a lognormal distribution. The proportion of male was compared between groups with a Chi-square test and continuous baseline characteristics were compared using a Student's $t$-test. All measurements including parameters of cardiopulmonary function, biomarkers, and arterial blood flow were analyzed using ANCOVA or repeated measures ANCOVA including age as a covariate to control for its potentially confounding effect. Contrasts between groups were performed at each time point in the repeated measures model. Basal and hyperemic arterial blood flows were summarized by computing area under the curve. Results are expressed as adjusted means \pm standard errors or adjusted geometric means. To evaluate whether biomarkers influenced aerobic capacity, Pearson's correlations were performed. A $P$ value $<0.05$ was considered statistically significant. Statistical analyses were performed using the SAS software (version 9.2 or higher).

\section{Results}

A total of 32 subjects were recruited for this study including 18 patients with HFpEF and 14 healthy subjects. The clinical characteristics of the study population are shown in Table 1. The majority of patients exhibited systemic hypertension as a cause of HF. Of the patients studied, $83 \%$ were in NYHA class II symptoms at the time of admission. All HFpEF patients exhibited a larger LV end-diastolic volume and a shorter peak filling rate (PFR) with a higher time to PFR compared with the healthy subjects confirming a significant diastolic dysfunction in our patients. LVEF was higher in patients with HFpEF. The majority of patients (67\%) were treated with 
TABLE 2: Exercise haemodynamics and gas exchange parameters for the study population.

\begin{tabular}{lcc}
\hline Stress variables & $\begin{array}{c}\text { HFpEF patients } \\
(n=18)\end{array}$ & $\begin{array}{c}\text { Healthy controls } \\
(n=14)\end{array}$ \\
\hline Duration (min) & $8.33 \pm 0.48^{*}$ & $10.36 \pm 0.55$ \\
Maximal energy expenditure (METS) & $4.81 \pm 0.21^{* * *}$ & $8.07 \pm 0.48$ \\
Peak exercise heart rate (bpm) & $106 \pm 5^{* * *}$ & $162 \pm 6$ \\
Peak exercise systolic blood pressure $(\mathrm{mmHg})$ & $158 \pm 6^{*}$ & $180 \pm 7$ \\
Peak exercise diastolic blood pressure $(\mathrm{mmHg})$ & $74.1 \pm 1.9$ & $79.6 \pm 2.2$ \\
Peak $\mathrm{VO}_{2}$ (mL/kg/min) & $12.0 \pm 0.44^{* * *}$ & $19.1 \pm 1.07$ \\
\% of $\mathrm{VO}_{2}$ predicted for age & $87 \pm 5^{* * *}$ & $123 \pm 6$ \\
Heart rate recovery at 1 min $(\mathrm{bpm})$ & $17.0 \pm 2.2^{*}$ & $24.4 \pm 2.6$ \\
Heart rate recovery at 2 min $(\mathrm{bpm})$ & $32.1 \pm 3.1^{* * *}$ & $50.0 \pm 3.6$ \\
VE/VCO slope & $33.6^{*}$ & 29.3 \\
OUES & $1.55 \pm 0.12^{*}$ & $2.06 \pm 0.14$ \\
\hline
\end{tabular}

METS: metabolic equivalent tasks; OUES: oxygen uptake efficiency slope; $\mathrm{VCO}_{2}$ : exhale carbon dioxide; VE: ventilation; $\mathrm{VO}_{2}$ : oxygen uptake. Values are expressed as adjusted mean \pm standard error or adjusted geometric mean. ${ }^{*} P<0.05 ;{ }^{* * *} P<0.001$. For the $\mathrm{VE} / \mathrm{VCO}_{2}$ slope variable, there was a significant interaction age $*$ group. In this table, we present the adjusted geometric means for an age of 68 years (median value) which is the closest age compared with our HFpEF patients. For Q1 (61 year old), there was no significant difference between HFpEF patients and healthy control subjects $(30.9$ versus $29.7, P=0.57)$. For Q3 (75 year old), there was a significant difference between HFpEF and healthy control subjects (36.7 versus $28.9, P<0.01$ ).

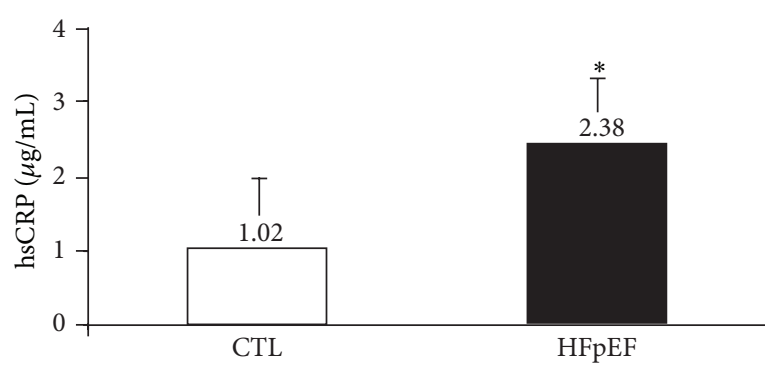

(a)

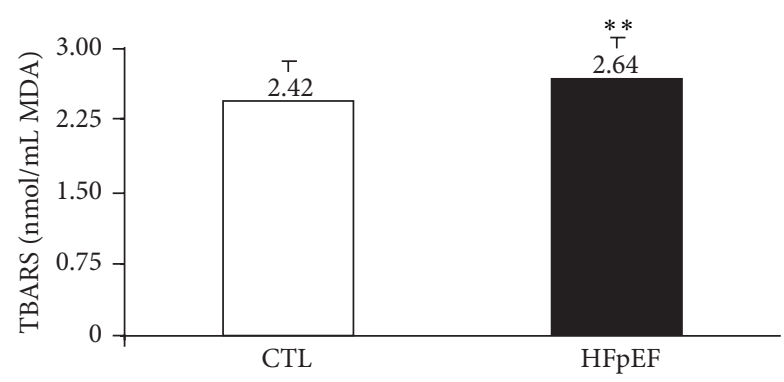

(c)

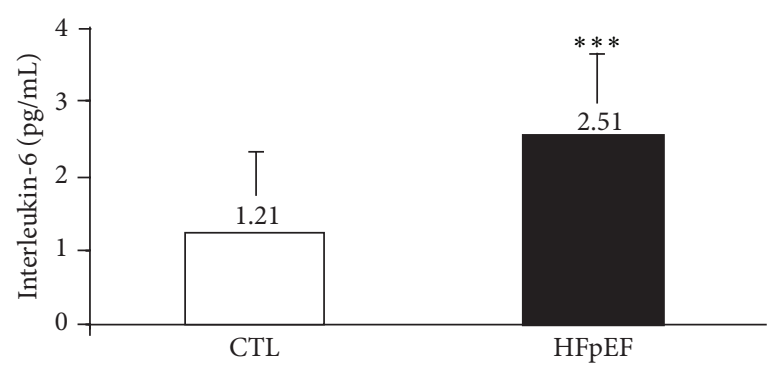

(b)

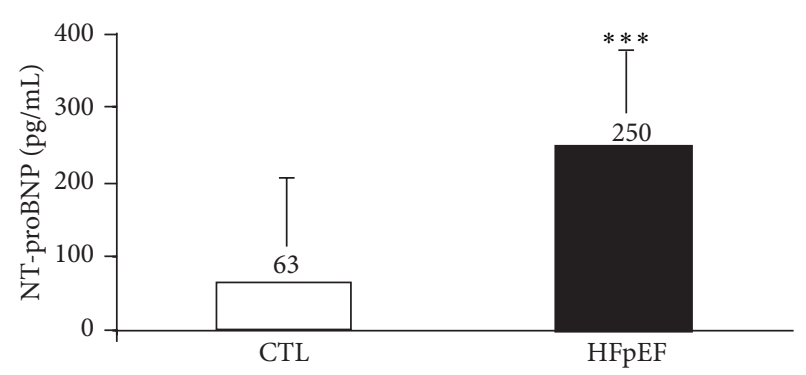

(d)

FIGURE 1: Circulating biomarker levels for patients with HFpEF versus healthy control subjects. NT-proBNP: N-terminal prohormone of brain natriuretic peptide; hsCRP: high-sensitivity C-reactive protein; TBARS: thiobarbituric acid reactive substances. Values are expressed as adjusted geometric mean or adjusted mean \pm error. Significantly different from HFpEF values: ${ }^{*} P<0.05 ;{ }^{* *} P<0.01 ;{ }^{* * *} P<0.001$.

an angiotensin II receptor blocker (ARBs) and 50\% received a beta-blocker.

Exercise and gas exchange parameters are presented in Table 2. All patients and healthy subjects performed a maximal effort as evidenced by a respiratory exchange ratio $>1.05$ (data not shown). Exercise duration and peak METS achieved were significantly lower in patients with HFpEF compared with healthy subjects. The OUES was reduced by $31 \%$ in our patients. Similarly, peak $\mathrm{VO}_{2}$ and the $\mathrm{VE} / \mathrm{VCO}_{2}$ slope were significantly decreased by $41 \%$ and increased by $15 \%$, respectively. HRR at 1 and 2 min after the termination of exercise were significantly lower in patients compared with the healthy subjects.

Biomarkers data for the study population are presented in Figures 1 and 2. Plasma levels of hsCRP $(P<0.05)$, TBARS $(P<0.01)$, and 8 -epi-prostaglandin F2 $\alpha(P<0.05)$ were significantly increased in patients with HFpEF compared with healthy subjects. The patients exhibited a 4-fold 
TABLE 3: Correlations between biomarkers and peak $\mathrm{VO}_{2}$ for the study population.

\begin{tabular}{|c|c|c|c|c|c|c|c|}
\hline & Peak $\mathrm{VO}_{2}$ & HRR 2 & $\mathrm{BNP}$ & hsCRP & IL-6 & 8-epi-PG-F2 $\alpha$ & TBARS \\
\hline \multicolumn{8}{|c|}{ Pearson correlation coefficients ( $P$ values $)$} \\
\hline Peak $\mathrm{VO}_{2}$ & 1 & & & & & & \\
\hline HRR 2 & $0.71^{* * *}$ & 1 & & & & & \\
\hline BNP & $-0.66^{* * *}$ & $-0.57^{* * *}$ & 1 & & & & \\
\hline hsCRP & $-0.40^{*}$ & $-0.49^{* *}$ & $0.45^{*}$ & 1 & & & \\
\hline IL-6 & $-0.63^{* * *}$ & $-0.57^{* * *}$ & $0.70^{* * *}$ & $0.67^{* * *}$ & 1 & & \\
\hline 8 -epi-PG-F $F_{2 \alpha}$ & $-0.41^{*}$ & $-0.38^{*}$ & $0.44^{*}$ & 0.21 & $0.55^{* *}$ & 1 & \\
\hline TBARS & -0.22 & -0.19 & 0.26 & $0.39^{*}$ & $0.41^{*}$ & $0.43^{*}$ & 1 \\
\hline
\end{tabular}

BNP: brain natriuretic peptide; HRR2: heart rate recovery at 2 min following the end of exercise; hsCRP: high-sensitivity C-reactive protein; IL-6: interleukin6; TBARS: thiobarbituric acid reactive substances; $\mathrm{VO}_{2}$ : oxygen consumption; 8-epi-PG-F2 $\alpha$ : 8-epi-prostaglandin $\mathrm{F} 2 \alpha .{ }^{*} P<0.05 ;{ }^{* *} P<0.01 ;{ }^{* * *} P<0.001$.

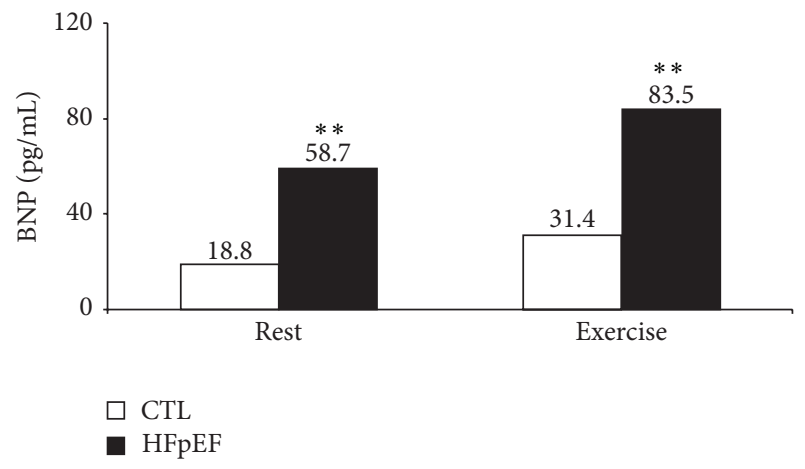

FIGURE 2: Changes in brain natriuretic peptide at rest and at peak exercise in patients with HFpEF versus healthy subjects. BNP: brain natriuretic peptide. Values are expressed as adjusted geometric mean. Significantly different from HFpEF values: ${ }^{* *} P<0.01$.

increase in NT-proBNP $(P<0.001)$ (Figure 1) and a 3-fold increase in BNP plasma concentrations $(P<0.01)$ in resting state (Figure 1). This difference persisted at peak exercise (Figure 2).

The relationships between biomarkers with selected exercise and biochemistry parameters are presented in Table 3 and Figure 3. Significant relationships were observed between BNP, hsCRP, IL-6, and 8-epi-prostaglandin F2 $\alpha$ and peak $\mathrm{VO}_{2}$ and HRR 2 (Table 3). There was also a modest but significant relationship between hsCRP and IL- 6 and between hsCRP and exercise duration in the HFpEF population (Figure 3).

Peripheral arterial flows in resting state and following arterial occlusion are presented in Figure 4. Basal peripheral arterial forearm blood flow was not statistically different in the study population as demonstrated by the area under the curve (AUC) in HFpEF patients compared with healthy subjects (resp., $523 \pm 70$ versus $386 \pm 41$, NS) (Figure 4(a)). No difference in the hyperemic response was observed between the two groups (Figure 4(b)).

\section{Discussion}

In this study we reported a significant reduction in aerobic capacity and oxygen uptake efficiency in ambulatory patients with HFpEF. We also reported a significant increase in some biomarkers related to subclinical inflammation and oxidative stress. Both BNP and NT-proBNP were significantly elevated at rest with a similar magnitude of BNP increase at peak exercise in both patients and healthy subjects. In addition, we observed some significant relationship between peak aerobic capacity and HRR following exercise with BNP, IL6 , and 8-epi-prostaglandin F2 $\alpha$. We observed no significant differences in basal and posthyperemic blood flow in HFpEF patients compared with healthy subjects.

Previous investigations have reported a significant reduction in functional and peak aerobic capacities in patients with HFpEF [2, 18-20]. Here we reported a decrease in peak $\mathrm{VO}_{2}$ of $37 \%$ in patients with HFpEF compared with controls. This magnitude of decrease is in agreement with the overall decrease of $40 \%$ reported by other investigators [2, 18-20]. In addition, we observed a $30 \%$ reduction in the OUES in HFpEF patients compared with healthy control subjects. These changes are consistent with previous reports $[2,20]$ showing significant decrease in cardiopulmonary reserve and abnormal ventilator function in these patients.

Previous investigations have shown an increase in selected biomarkers such as IL-6 and NT-proBNP in patients with $\operatorname{HFpEF}[7,8,21]$. Our findings confirm our former observations and data from other investigators showing significant increases of the C-reactive protein and IL-6 and demonstrating a significant proinflammatory state in these patients $[7,21,22]$. In addition to earlier studies [23, 24], we reported a 3-fold increase in BNP at rest which was maintained at peak exercise in HFpEF patients. The similar magnitude of BNP increase at peak exercise for both HF and health subjects patients suggests a preservation of wall stress during exercise in patients with HFpEF. Here we also reported a significant increase in biomarkers related to oxidative stress in patients with HFpEF compared with healthy subjects. These findings have not been reported before. Indeed, two biomarkers of oxidative stress including TBARS and 8 -epi-prostaglandin F2 $\alpha$ were both significantly increased, confirming a prooxidative state in these patients. Previous investigations have reported a role of oxidative stress in the pathophysiology of HF $[25,26]$. Other observations have reported a detrimental effect of oxidative stress on the degradation of cardiac extracellular matrix degradation in 


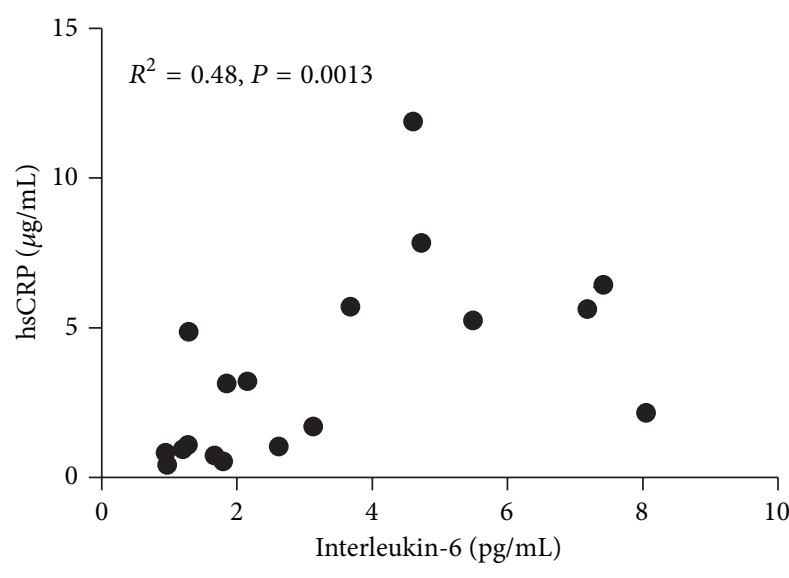

(a)

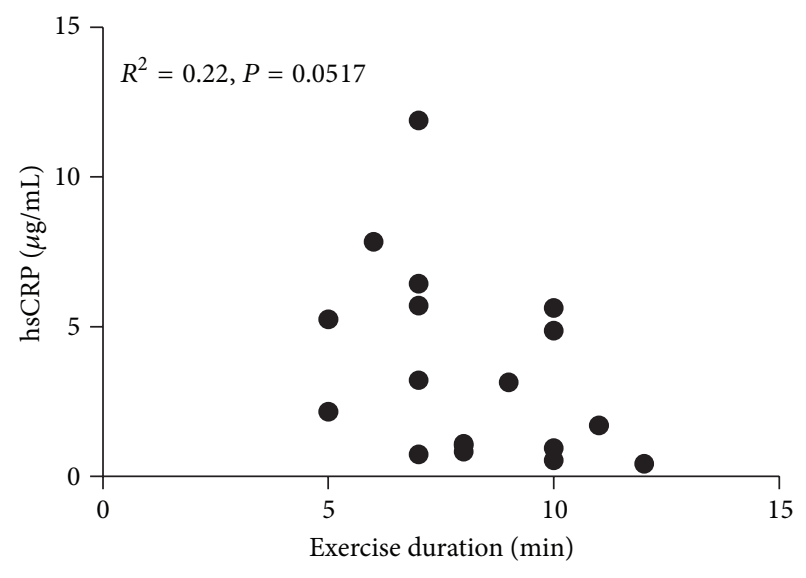

(b)

FIGURE 3: Relationships between selected inflammatory biomarkers and exercise duration in patients with HFpEF. hsCRP: high-sensitivity C-reactive protein; $R^{2}$ : coefficient of determination.

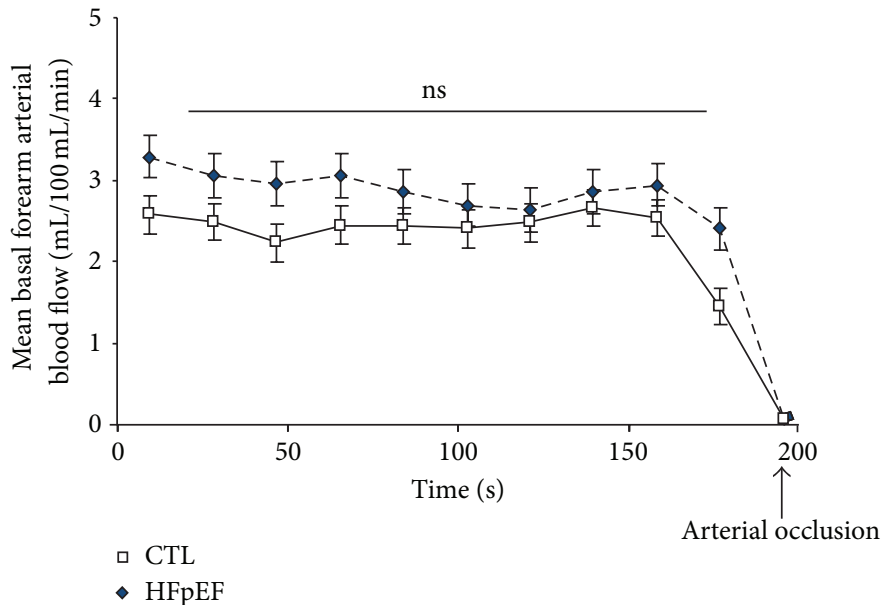

(a)

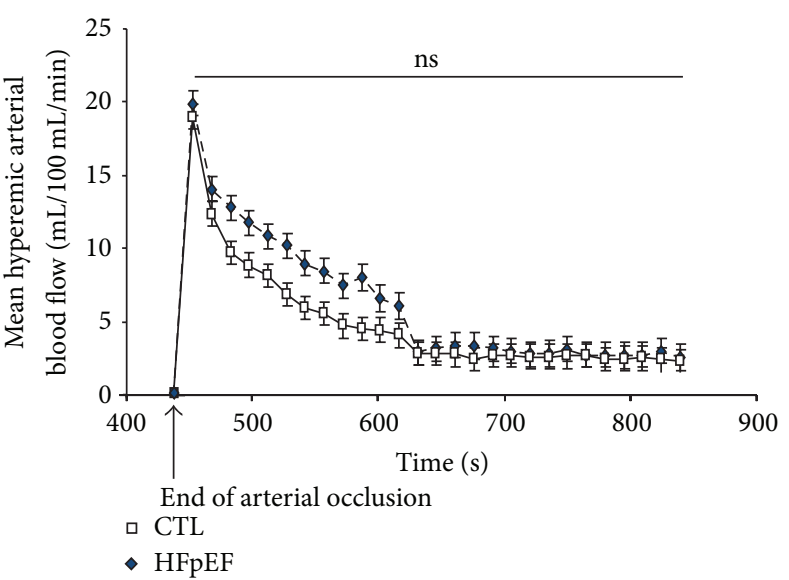

(b)

FIGURE 4: Changes in basal (a) and hyperemic (b) arterial blood flow for patients with HFpEF versus healthy control subjects. Values are expressed as adjusted mean \pm standard error.

humans [27] and on the cardiac contractility in mice [28]. The role of biomarker changes and specially those related to subclinical inflammation and oxidative stress on the pathophysiology of HFpEF remain unknown. We further explored the relationships between selected clinical and functional parameters with some biomarkers in our study population. We reported a significant relationship between peak $\mathrm{VO}_{2}$ and HRR at 2 minutes with BNP, 8-epi-prostaglandin F2 $\alpha$, hsCRP, and IL- 6 in the overall population. This suggests a significant relationship between inflammation and autonomic regulation with functional capacity in HFpEF patients. These observations are in agreement with previous studies showing a relationship between sympathetic and parasympathetic tones and regulation of inflammation in chronic HF patients [29] and in a canine pacing model of HF [30]. Additional investigations are needed to confirm these findings.
Here, we reported no significant differences in basal and posthyperemic peripheral arterial blood flow in patients with HFpEF compared with healthy subjects. Abnormal endothelial function is associated with a decreased aerobic capacity in high risk patients [9] and in patients with HF with decreased LVEF [12]. There has been little data regarding the changes in peripheral arterial blood flow at rest and following stress in patients with HFpEF. A previous investigation reported a decrease in leg blood flow at rest and following exercise [1]. In contrast, other clinical studies reported no difference in leg flow-mediated dilation [11] or in brachial artery flow-mediated dilation [10] following submaximal exercise compared with healthy subjects. In that same study, no significant relationship between the reduction in peak $\mathrm{VO}_{2}$ and brachial artery flow-mediated dilation has been reported beyond the effect of aging [10]. The differences 
between a previous study [1] and our data may be explained by some clinical differences in the patient population and methodological approaches. First, the etiology of HF was different with some patients presenting dyspnea because of bronchial asthma in the latter study [1]. Most importantly, the rate of use of angiotensin-II modulating agents was $73 \%$ in the current study as opposed to $40 \%$ on average in previous publications $[1,10]$. The high proportion of use of ARBs (i.e., 67\%) may have contributed to attenuate the changes in basal and posthyperemic blood flow in our patients [31, 32]. Finally, we used SGP as opposed to magnetic resonance $[1,11]$ or brachial artery flow-mediated dilation $[10]$ methods. Contrary to these techniques, we mechanically assessed the increase in forearm volume after the cuff deflation using calibrated strain gauges connected to a plethysmograph. This technique correlates well with the near-infrared spectroscopy for noninvasive assessment of arterial forearm flow [17]. Nevertheless SGP may not be sensitive enough to detect small changes in microvascular function in HFpEF patients.

Several factors may limit the conclusions of this study. Firstly, the population of patients was older than the control population. However, to minimize the impact of age on our observations ANCOVA analyses were computed using age as a covariate. Also no investigations have reported any effect of age on biomarkers and functional parameters in patients with symptomatic HF caused by preserved ejection fraction. Secondly, the sample size was small. Despite this, our study population was fairly homogenous allowing small variance and significance in most of the parameters studied. Thirdly chronic use of ARBs may have significantly impacted our findings on forearm blood flow data. Finally, we only measured plasma level of BNP at peak exercise. The inclusion of other biomarkers may have provided additional insights on the mechanisms involved with exercise limitations in these patients.

In conclusion, this study demonstrates that ambulatory patients with HFpEF exhibit a significant reduction in cardiopulmonary reserve and oxygen uptake efficiency concomitantly with an elevation in broad spectrum of biomarkers confirming a proinflammatory and a prooxidative status in these patients. The relationship between some biomarkers of inflammation and oxidative stress suggest a role of these processes on functional capacity in these patients. The role of biomarkers and the assessment of peripheral arterial function by multimodality techniques deserve further investigations.

\section{Conflict of Interests}

The authors declare that there is no conflict of interests regarding the publication of this paper.

\section{Disclosure}

The study is funded by a grant-in-aid from the Heart and Stroke Foundation of Canada. Michel White holds the Carolyn and Richard Renaud Research Chair in heart failure of the Montreal Heart Institute. Simon de Denus holds the Beaulieu-Saucier chair in pharmacogenomics of the Université de Montréal.

\section{Acknowledgment}

The authors are grateful to the superb work provided by the secretarial team at the Montreal Heart Institute.

\section{References}

[1] C. Puntawangkoon, D. W. Kitzman, S. B. Kritchevsky et al., "Reduced peripheral arterial blood flow with preserved cardiac output during submaximal bicycle exercise in elderly heart failure," Journal of Cardiovascular Magnetic Resonance, vol. 11, no. 1, p. 48, 2009.

[2] P. S. Bhella, A. Prasad, K. Heinicke et al., "Abnormal haemodynamic response to exercise in heart failure with preserved ejection fraction," European Journal of Heart Failure, vol. 13, no. 12, pp. 1296-1304, 2011.

[3] M. Hollenberg and I. B. Tager, "Oxygen uptake efficiency slope: an index of exercise performance and cardiopulmonary reserve requiring only submaximal exercise," Journal of the American College of Cardiology, vol. 36, no. 1, pp. 194-201, 2000.

[4] C. Van Laethem, J. Bartunek, M. Goethals, P. Nellens, E. Andries, and M. Vanderheyden, "Oxygen uptake efficiency slope, a new submaximal parameter in evaluating exercise capacity in chronic heart failure patients," American Heart Journal, vol. 149, no. 1, pp. 175-180, 2005.

[5] L. C. Davies, R. Wensel, P. Georgiadou et al., "Enhanced prognostic value from cardiopulmonary exercise testing in chronic heart failure by non-linear analysis: oxygen uptake efficiency slope," European Heart Journal, vol. 27, no. 6, pp. 684-690, 2006.

[6] R. Arena, P. Brubaker, B. Moore, and D. Kitzman, "The oxygen uptake efficiency slope is reduced in older patients with heart failure and a normal ejection fraction," International Journal of Cardiology, vol. 144, no. 1, pp. 101-102, 2010.

[7] S. de Denus, J. Lavoie, A. Ducharme et al., "Differences in biomarkers in patients with heart failure with a reduced vs a preserved left ventricular ejection fraction," Canadian Journal of Cardiology, vol. 28, no. 1, pp. 62-68, 2012.

[8] M. Niethammer, M. Sieber, S. von Haehling et al., "Inflammatory pathways in patients with heart failure and preserved ejection fraction," International Journal of Cardiology, vol. 129, no. 1, pp. 111-117, 2008.

[9] A. Clark, M. Volterrani, J. W. Swan, D. Hue, J. Hooper, and A. J. S. Coats, "Leg blood flow, metabolism and exercise capacity in chronic stable heart failure," International Journal of Cardiology, vol. 55, no. 2, pp. 127-135, 1996.

[10] M. J. Haykowsky, D. M. Herrington, P. H. Brubaker, T. M. Morgan, W. G. Hundley, and D. W. Kitzman, "Relationship of flow-mediated arterial dilation and exercise capacity in older patients with heart failure and preserved ejection fraction," Journals of Gerontology A, vol. 68, no. 2, pp. 161-167, 2013.

[11] W. G. Hundley, E. Bayram, C. A. Hamilton et al., "Leg flowmediated arterial dilation in elderly patients with heart failure and normal left ventricular ejection fraction," American Journal of Physiology, vol. 292, no. 3, pp. H1427-H1434, 2007.

[12] B. Meyer, D. Mörtl, K. Strecker et al., "Flow-mediated vasodilation predicts outcome in patients with chronic heart failure: comparison with B-type natriuretic peptide," Journal of the 
American College of Cardiology, vol. 46, no. 6, pp. 1011-1018, 2005.

[13] J. Myers, D. Walsh, N. Buchanan, P. McAuley, E. Bowes, and V. Froelicher, "Increase in blood lactate during ramp exercise: comparison of continuous and threshold models," Medicine and Science in Sports and Exercise, vol. 26, no. 11, pp. 1413-1419, 1994.

[14] E. Normandin, A. Nigam, P. Meyer et al., "Acute responses to intermittent and continuous exercise in heart failure patients," Canadian Journal of Cardiology, vol. 29, no. 4, pp. 466-471, 2013.

[15] R. Baba, M. Nagashima, M. Goto et al., "Oxygen uptake efficiency slope: a new index of cardiorespiratory functional reserve derived from the relation between oxygen uptake and minute ventilation during incremental exercise," Journal of the American College of Cardiology, vol. 28, no. 6, pp. 1567-1572, 1996.

[16] A. Virdis, M. F. Neves, F. Amiri, E. Viel, R. M. Touyz, and E. L. Schiffrin, "Spironolactone improves angiotensin-induced vascular changes and oxidative stress," Hypertension, vol. 40, no. 4, pp. 504-510, 2002.

[17] F. Harel, N. Olamaei, Q. Ngo, J. Dupuis, and P. Khairy, "Arterial flow measurements during reactive hyperemia using NIRS," Physiological Measurement, vol. 29, no. 9, pp. 1033-1040, 2008.

[18] B. A. Borlaug, V. Melenovsky, S. D. Russell et al., "Impaired chronotropic and vasodilator reserves limit exercise capacity in patients with heart failure and a preserved ejection fraction," Circulation, vol. 114, no. 20, pp. 2138-2147, 2006.

[19] T. T. Phan, K. Abozguia, G. Nallur Shivu et al., "Heart failure with preserved ejection fraction is characterized by dynamic impairment of active relaxation and contraction of the left ventricle on exercise and associated with myocardial energy deficiency," Journal of the American College of Cardiology, vol. 54, no. 5, pp. 402-409, 2009.

[20] T. T. Phan, G. N. Shivu, K. Abozguia et al., "Impaired heart rate recovery and chronotropic incompetence in patients with heart failure with preserved ejection fraction," Circulation, vol. 3, no. 1, pp. 29-34, 2010.

[21] K. Bishu, A. Deswal, H. H. Chen et al., "Biomarkers in acutely decompensated heart failure with preserved or reduced ejection fraction," American Heart Journal, vol. 164, no. 5, pp. 763-770, 2012.

[22] A. Kalogeropoulos, V. Georgiopoulou, B. M. Psaty et al., "Inflammatory markers and incident heart failure risk in older adults. The health ABC (health, aging, and body composition) study," Journal of the American College of Cardiology, vol. 55, no. 19, pp. 2129-2137, 2010.

[23] B. A. Borlaug, T. P. Olson, C. S. P. Lam et al., "Global cardiovascular reserve dysfunction in heart failure with preserved ejection fraction," Journal of the American College of Cardiology, vol. 56, no. 11, pp. 845-854, 2010.

[24] C. Tschöpe, M. Kašner, D. Westermann, R. Gaub, W. C. Poller, and H. Schultheiss, "The role of NT-proBNP in the diagnostics of isolated diastolic dysfunction: correlation with echocardiographic and invasive measurements," European Heart Journal, vol. 26, no. 21, pp. 2277-2284, 2005.

[25] S. F. Mohammed, T. Ohtani, J. Korinek et al., "Mineralocorticoid accelerates transition to heart failure with preserved ejection fraction via 'nongenomic effects', Circulation, vol. 122, no. 4, pp. 370-378, 2010.

[26] E. Braunwald, "Medical progress: biomarkers in heart failure," New England Journal of Medicine, vol. 358, no. 20, pp. 20942159, 2008.
[27] H. Nagaset and J. F. Woessner Jr., "Matrix metalloproteinases," Journal of Biological Chemistry, vol. 274, no. 31, pp. 21491-21494, 1999.

[28] M. Cox, U. A. Hawkins, B. D. Hoit, and S. C. Tyagi, "Attenuation of oxidative stress and remodeling by cardiac inhibitor of metalloproteinase protein transfer," Circulation, vol. 109, no. 17, pp. 2123-2128, 2004.

[29] J. R. Gage, G. Fonarow, M. Hamilton, M. Widawski, O. Martínez-Maza, and D. L. Vredevoe, "Beta blocker and angiotensin-converting enzyme inhibitor therapy is associated with decreased Th1/Th2 cytokine ratios and inflammatory cytokine production in patients with chronic heart failure," NeuroImmunoModulation, vol. 11, no. 3, pp. 173-180, 2004.

[30] Y. Zhang, Z. B. Popović, S. Bibevski et al., "Chronic vagus nerve stimulation improves autonomic control and attenuates systemic inflammation and heart failure progression in a canine high-rate pacing model," Circulation, vol. 2, no. 6, pp. 692-699, 2009.

[31] N. Preumont, P. Unger, S. Goldman, and G. Berkenboom, "Effect of long-term angiotensin II type I receptor antagonism on peripheral and coronary vasomotion," Cardiovascular Drugs and Therapy, vol. 18, no. 3, pp. 197-202, 2004.

[32] A. Warnholtz, M. A. Ostad, T. Heitzer et al., "AT1-receptor blockade with irbesartan improves peripheral but not coronary endothelial dysfunction in patients with stable coronary artery disease," Atherosclerosis, vol. 194, no. 2, pp. 439-445, 2007. 


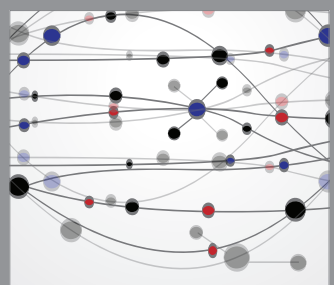

The Scientific World Journal
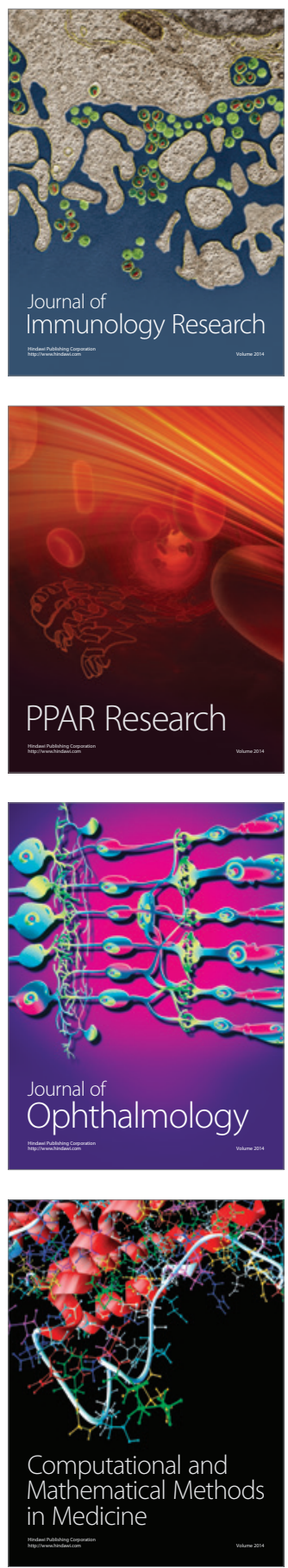

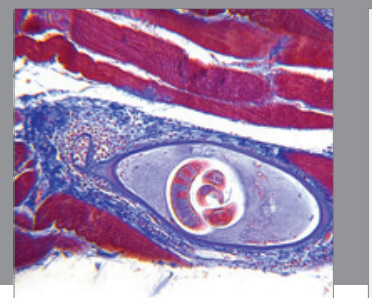

Gastroenterology

Research and Practice
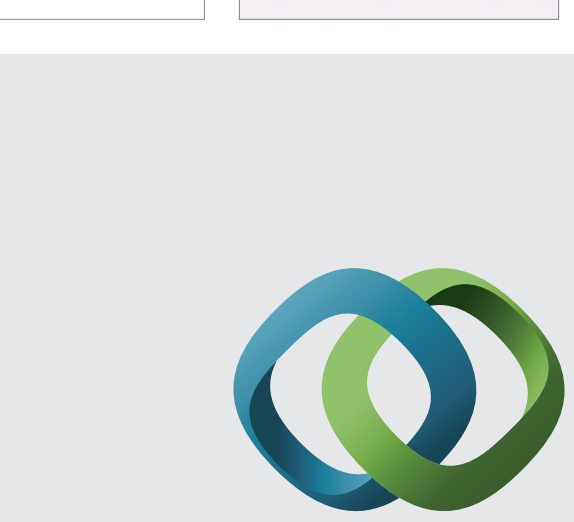

\section{Hindawi}

Submit your manuscripts at

http://www.hindawi.com
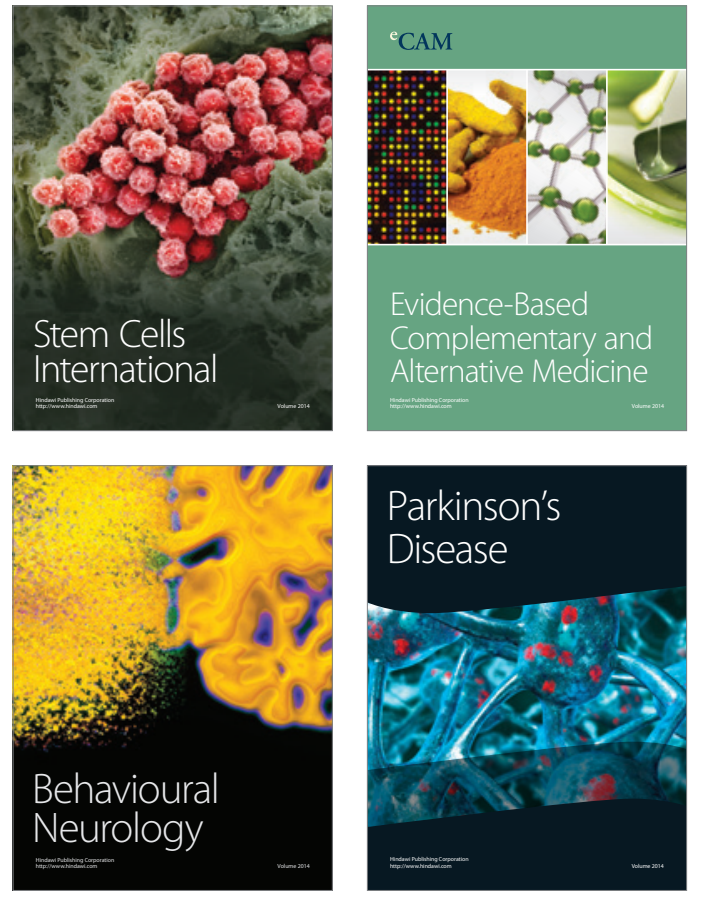
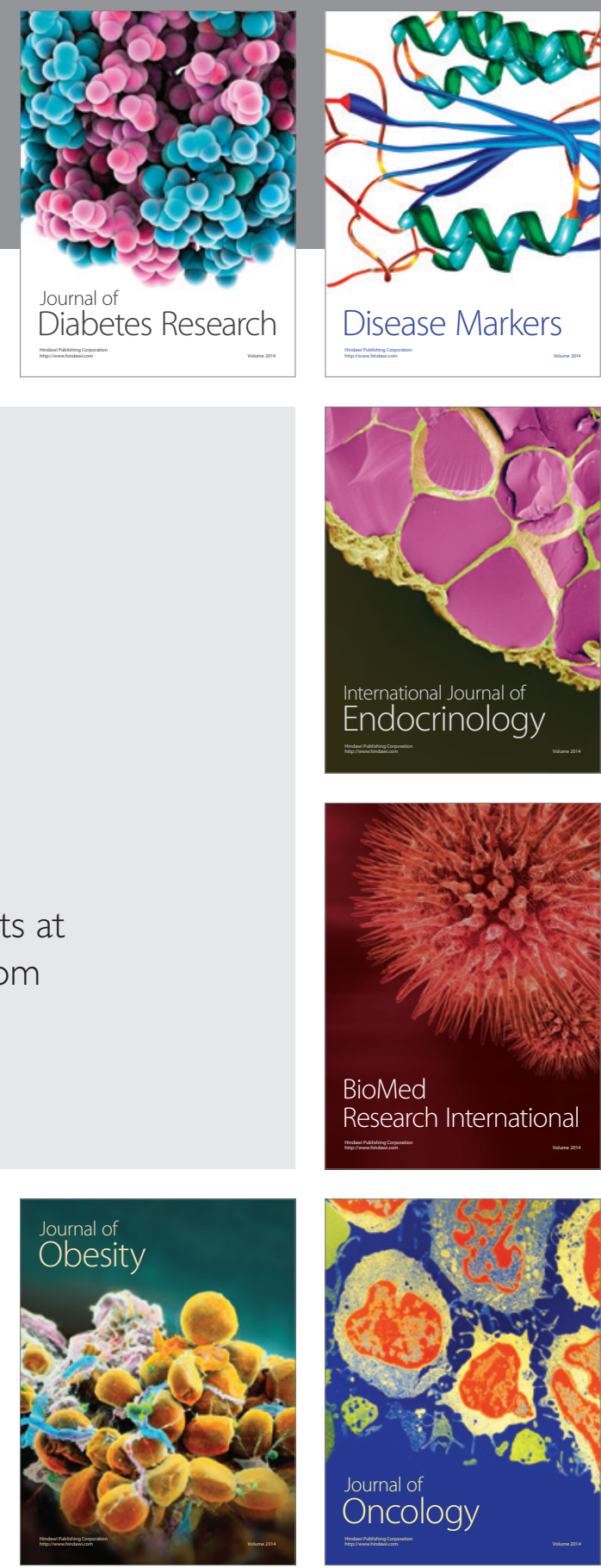

Disease Markers
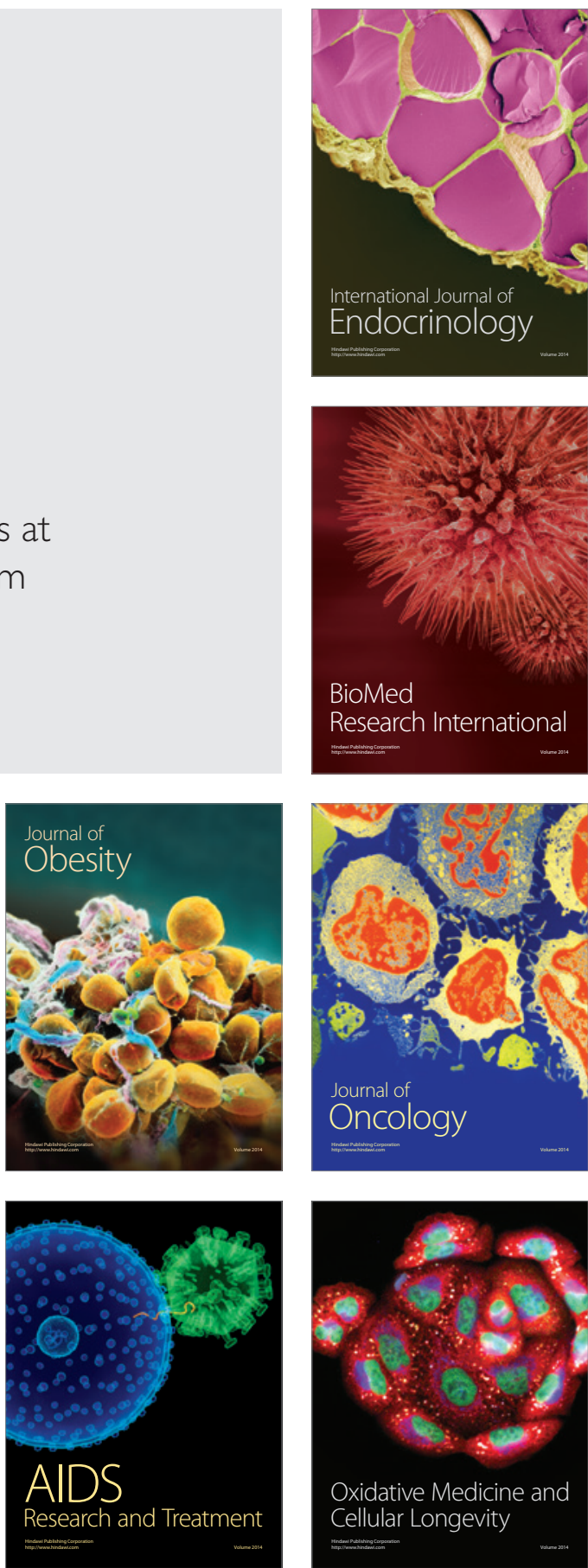